Supporting Information

\title{
Exposing Small-molecule Nano-entities By a Nuclear Magnetic Resonance Relaxation Assay
}

Yann Ayotte, ${ }^{1 \ddagger}$ Victoria M. Marando, ${ }^{2 \ddagger}$ Louis Vaillancourt, ${ }^{2}$ Patricia Bouchard,${ }^{2}$ Gregory Heffron, ${ }^{3}$ Paul W. Coote, ${ }^{2,3}$ Sacha T. Larda, ${ }^{*}, 2$ and Steven R. LaPlante ${ }^{*}, 1,2,3$

1 INRS-Institut Armand-Frappier Research Centre, 531 Boulevard des Prairies, Laval, Québec, H7V 1B7, CANADA

2 NMX Research and Solutions, Inc., 500 Boulevard Cartier Ouest, Laval, Québec, H7V 5B7, CANADA

${ }^{3}$ Harvard Medical School, 240 Longwood Ave., Boston, MA 02115, USA

¥ These authors contributed equally to this work

* Corresponding authors

Table of Contents:

1) Quantification of peak area and relaxation decay rates

2) Control non-aggregator and known aggregators in the NMR dilution assay

3) Sensitivity for aggregates of small molecules

4) Additional series of structurally related compounds with distinct T2-CPMG profiles

5) Consistency of T2-CPMG at other compound concentrations

6) Evidence for the detection of chemical exchange

7) Consistency between the use of T2 values and peak area percentages

8) Compound information 


\section{1) Quantification of peak area and relaxation decay rates}

CPMG spectra for all compounds were read into Matlab (R2016b. The MathWorks, Inc. Natick, Massachusetts, United States) using the toolbox matNMR (http://matnmr.sourceforge.net/). Artificial line broadening of $3 \mathrm{~Hz}$ was added to each spectrum by convolving with a Lorentzian line shape kernel using the Matlab function 'conv'. The kernel was defined explicitly using the equation of an absorption-mode Lorentzian line shape. ${ }^{1}$ Line broadening was added to improve signal-tonoise ahead of numerical calculations.

Regions containing peaks were detected in each $1 \mathrm{~ms}$ delay CPMG spectrum by thresholding above the 96th percentile of spectrum amplitude (excluding a $0.3 \mathrm{ppm}$ window around the strong DMSO reference peak). Regions narrower than $0.007 \mathrm{ppm}$ were discarded as being due to spikes in the noise. Each contiguous region was then dilated by $0.05 \mathrm{ppm}$ to ensure that the tails of the peak were included. This used the 'imdilate' function from the Matlab Image Processing Toolbox with a unit-kernel of width 0.05 /(sweep width in ppm) ${ }^{*}$ (number of points), rounded to the nearest integer. In some cases, a cluster of nearby peaks (either a multiplet or closely spaced peaks) end up in the same region, and are therefore treated together in subsequent calculations. Due to the $0.05 \mathrm{ppm}$ dilation, the minimum space between peaks in separate regions is $0.1 \mathrm{ppm}$, and peaks closer together than that are treated as a single cluster or multiplet.

Amplitude in the first CPMG spectrum (1 ms delay) was calculated for each region by integrating the spectrum in that region using the 'sum' function in Matlab. For each region, the decrease in amplitude due to T2 decay in all CPMG spectra was found by projecting the $1 \mathrm{~ms}$ CPMG onto each subsequent CPMG spectrum. For this projection we used the 'l' (backslash or left matrix divide) function in Matlab. This is the solution $a(\Delta)$ in the least squares sense of the equation $C P M G(\Delta)=a(\Delta)^{*} C P M G(1)$, where $\Delta$ is the relaxation delay period, $C P M G(1)$ is the spectrum with $1 \mathrm{~ms}$ relaxation delay, $C P M G(\Delta)$ is a CPMG spectrum with another delay, and $a(\Delta)$ is scalar showing the decrease in intensity between the two spectra. This calculation is done separately for each region containing a peak.

The scalars $\mathrm{a}(\Delta)$ for $\Delta=1,25,50,100,200,300,500$, and $800 \mathrm{~ms}$ were fit to an exponential decay using the 'fit' function with option 'exp1' in Matlab, to estimate T2 decay rates for each region/peak in each spectrum. In high throughput applications, we instead only use spectra for $\Delta$ $=1$ and $\Delta=800 \mathrm{~ms}$. Scalars a $(\Delta)$ for $\Delta=800 \mathrm{~ms}$ were reported in the third column in Figures 6 , 7 , and $\mathrm{S} 3$. 


\section{2) Control non-aggregator and known aggregators in the NMR dilution assay}

The results obtained from the T2-CPMG assay for etodolac, methylene blue and Evans blue correlate with results obtained from a previously published NMR dilution-based aggregation assay. The NMR dilution-based assay flags aggregates based on characteristic changes in ligand spectra across a range of concentrations..$^{2-4}$ In this assay, etodolac exhibits the behavior expected for a non-aggregator: as compound concentration decreases, peak intensity decreases with no changes observed in peak shape, chemical shift or number of resonances (Figure S1A). In contrast, two previously reported aggregators, methylene blue and Evans blue, show the expected behavior of aggregating compounds (Figures S1B,C). As the concentration of both methylene blue and Evans blue decreases, there are systematic changes in chemical shift, number of peaks, or line broadening. These behaviors indicate that these compounds are selfassociating and forming aggregates at high concentrations, hindering their ability to freely tumble thus changing the molecular environment of the observed nuclei.

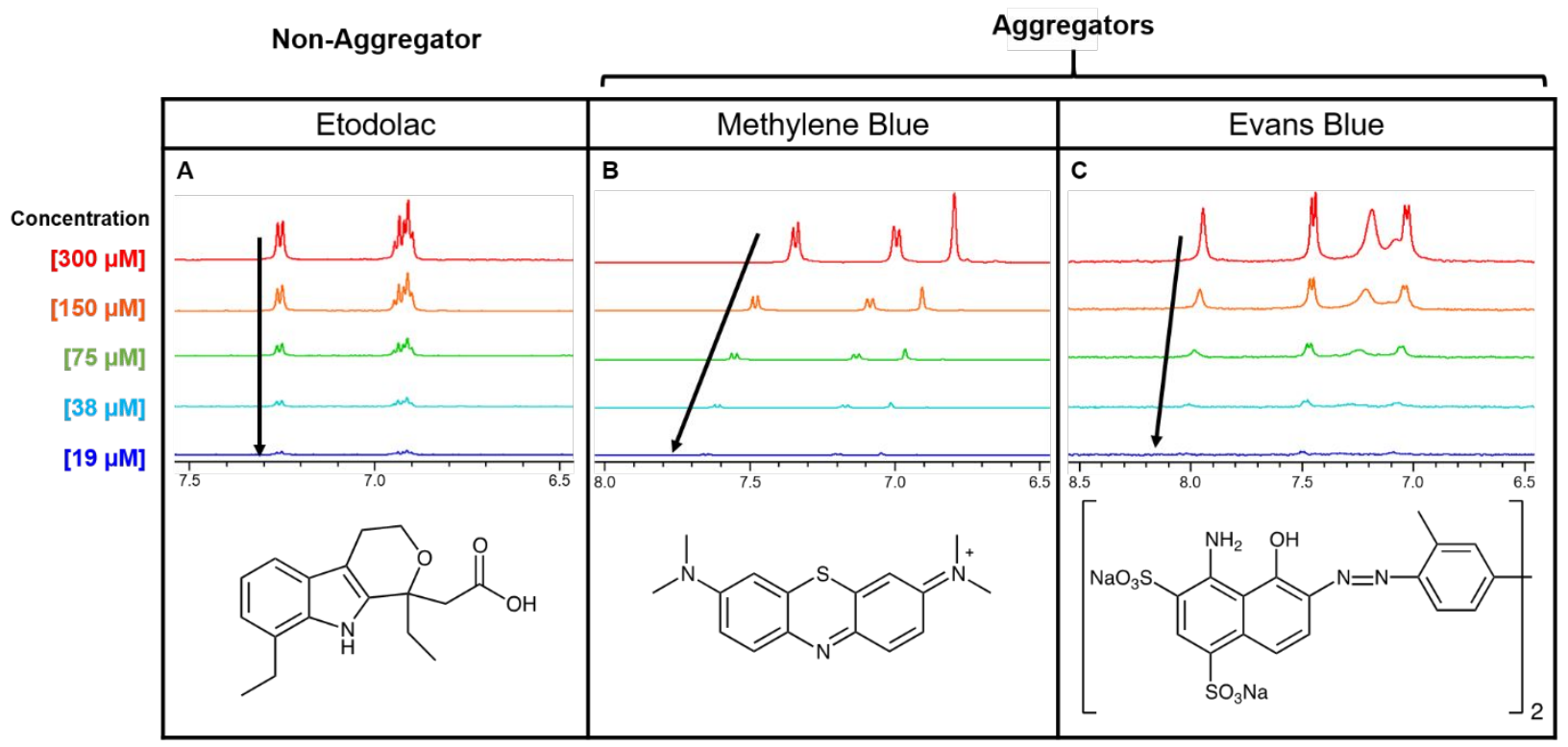

Figure S1. Dilution assay NMR spectra for three compounds illustrated in Figure 4. Panels A-C show a series of ${ }^{1} \mathrm{H}-\mathrm{NMR}$ spectra for concentrations ranging from 300 to $19 \mu \mathrm{M}$. The dilution profile supports the data obtained by the T2-CPMG aggregation assay.

\section{3) Sensitivity for aggregates of small molecules}

In the NMR dilution method, the control fragment (1), displays no significant changes in resonance number, shape and chemical shift (Figure S2A), consistent with the behavior of a nonaggregator. The T2-CPMG profile is also consistent with that of a non-aggregator. However, in the case of aggregating fragments 2 and 3, the T2-CPMG results from Figure 5 do not correlate with the observations made with the NMR dilution assay (Figure S2B,C). As compound 
concentration increases, there are no telltale signs of aggregation in the dilution assay and based solely on this assay, these compounds would have been cleared as non-aggregators. The T2CPMG assay clearly demonstrates that this is not the case.

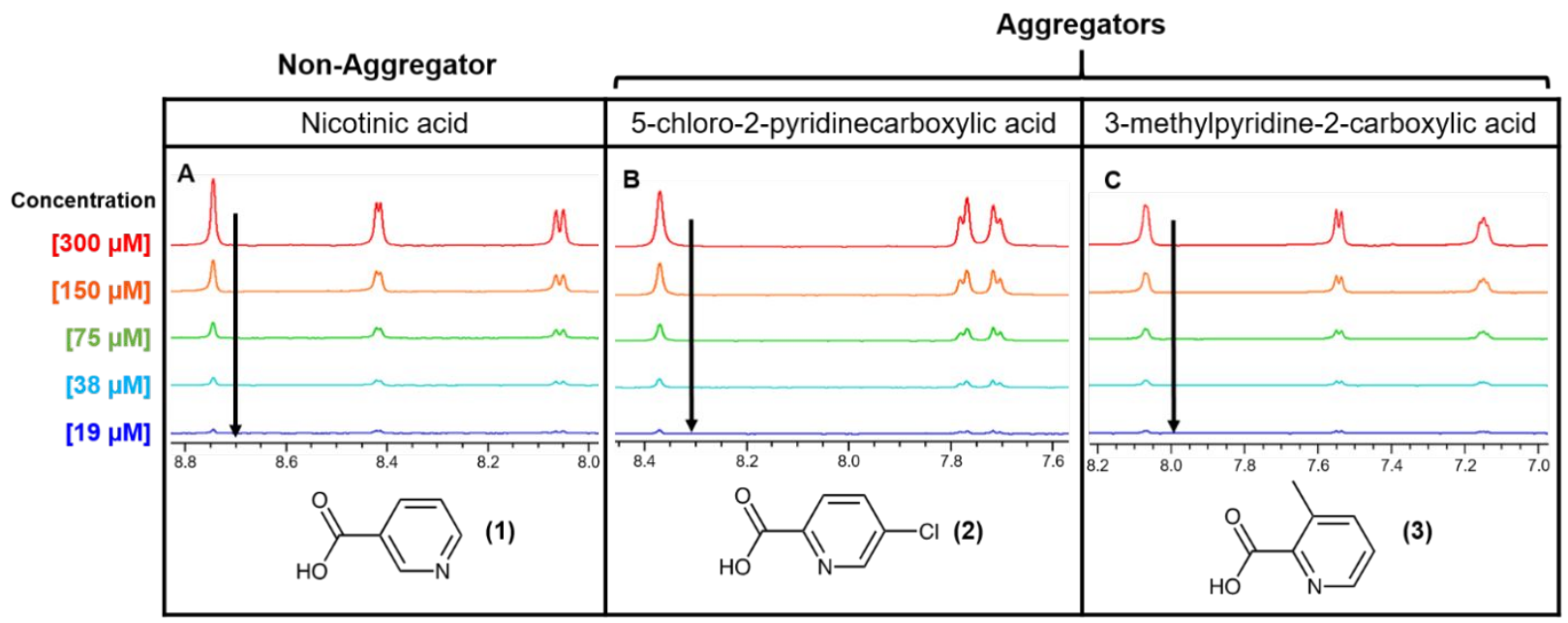

Figure S2. Dilution assay NMR spectra for three fragments illustrated in Figure 5. Panels A-C show a series of ${ }^{1} \mathrm{H}$-NMR spectra for concentrations ranging from 300 to $19 \mu \mathrm{M}$. While the dilution assay suggest that these compounds do not aggregate, the T2-CPMG assay indicates that these compounds undergo selfassociation.

\section{4) Additional series of structurally related compounds with distinct T2-CPMG profiles}

\begin{tabular}{|c|c|c|c|c|}
\hline Behavior & Structure & $\begin{array}{l}\text { Peak Chemical } \\
\text { Shift (6-10 ppm) }\end{array}$ & $\begin{array}{c}\text { Peak Area } \\
800 \mathrm{~ms} / 1 \mathrm{~ms} \text { T2-CPMG }\end{array}$ & NMR (aromatic region) \\
\hline \multirow{3}{*}{ Aggregator } & \multirow[b]{3}{*}{ (15) } & 6.83 & $41 \%$ & \\
\hline & & 7.05 & $12 \%$ & \\
\hline & & 7.40 & $15 \%$ & $8.0+7.5$ \\
\hline \multirow{3}{*}{ Aggregator } & \multirow{3}{*}{ (16) } & 7.16 & $34 \%$ & \\
\hline & & \multirow{2}{*}{$7.47-7.52$} & \multirow{2}{*}{$16 \%$} & \\
\hline & & & & $8.0 \quad 7.5$ \\
\hline \multirow{3}{*}{ Non-Aggregator } & \multirow[b]{3}{*}{ (17) } & 6.98 & $71 \%$ & \\
\hline & & 7.25 & $71 \%$ & \\
\hline & & 7.46 & $52 \%$ & $8.0+7.5$ \\
\hline \multirow{3}{*}{ Aggregator } & \multirow[b]{3}{*}{ (18) } & 6.96 & $6 \%$ & \\
\hline & & 7.20 & $0 \%$ & \\
\hline & & 7.43 & $4 \%$ & $8.0+7.5$ \\
\hline \multirow{3}{*}{ Non-Aggregator } & \multirow{3}{*}{ (19) } & 6.93 & $73 \%$ & \\
\hline & & 7.17 & $85 \%$ & $x$ \\
\hline & & 7.40 & $80 \%$ & $8.0+7.5$ \\
\hline
\end{tabular}

Figure S3. Quantification of a series of structurally related benzimidazole core compounds. For each compound, peaks in the aromatic region of the spectra $(6-10 \mathrm{ppm})$ are identified and the peak area was 
determined by integration. The peak areas between $1 \mathrm{~ms}$ and $800 \mathrm{~ms}$ delay times were compared and the percentage difference was calculated. These percentages can be compared to the NMR spectra in the far right column. The bottom, red spectrum has a $1 \mathrm{~ms}$ delay time and the top, brown spectrum has an $800 \mathrm{~ms}$ delay time.

\section{5) Consistency of T2-CPMG at other compound concentrations}

Although the compound concentrations used for T2-CPMG experiments throughout this manuscript have been $300 \mu \mathrm{M}$, the T2-CPMG assay can be used at various concentrations so long as there is sufficient NMR signal. Figure S4 compares the T2-CPMG profiles illustrated in Figure 4 at $300 \mu \mathrm{M}$ (Figure S4A-C) and the same experiments performed at $75 \mu \mathrm{M}$ compound concentration (Figure S4D-F). There is excellent agreement between the spectra observed between 300 and $75 \mu \mathrm{M}$, although it is highly conceivable that different results could be observed as a compound may aggregate at a higher concentration but not at a lower one.

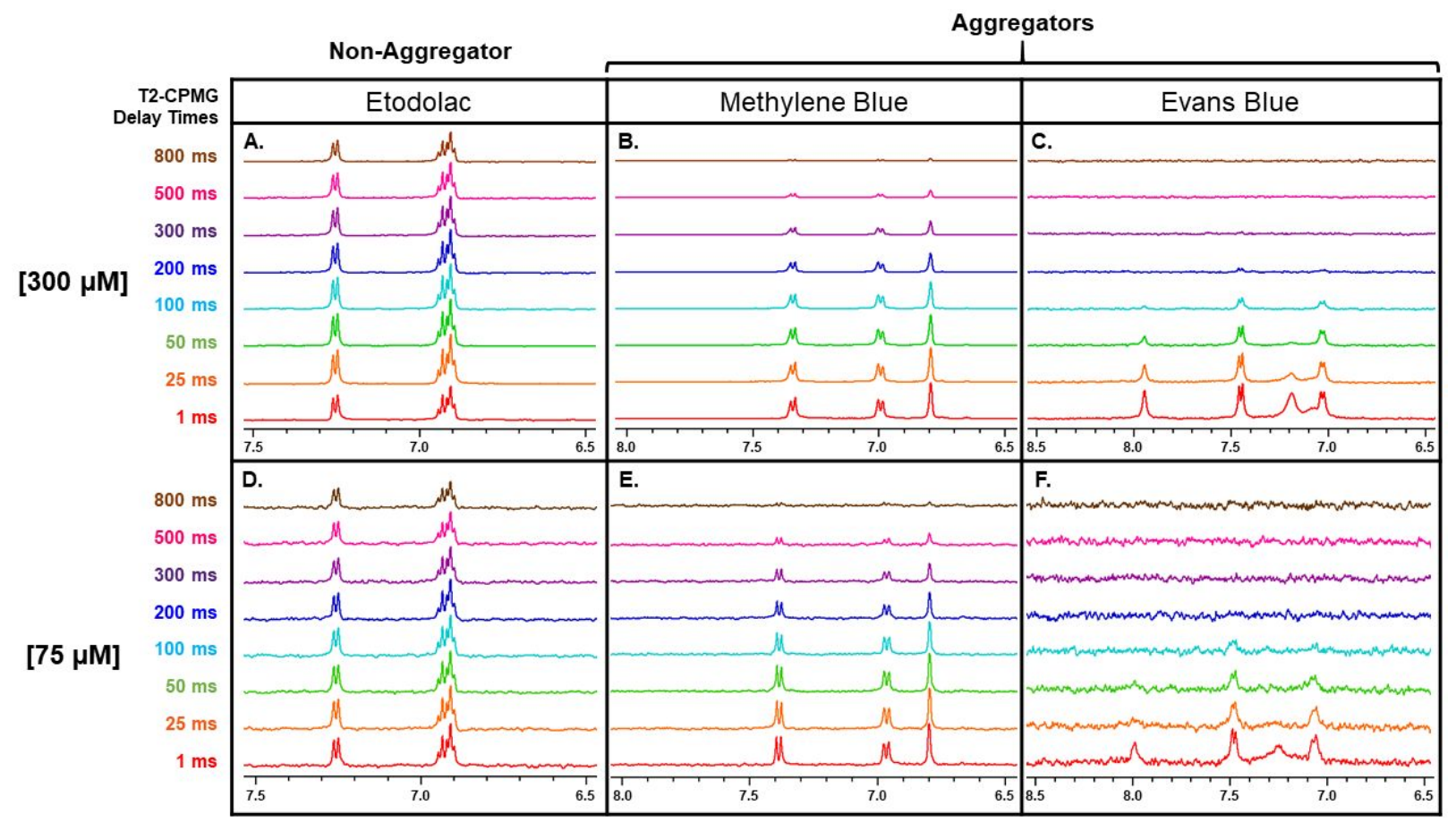

Figure S4. Shown are three examples demonstrating that the T2-CPMG assay can be run a different compound concentration. Panels A-C show a stack of the ${ }^{1} \mathrm{H}-\mathrm{NMR}$ spectra from various T2-CPMG delay times for a constant compound concentration of $300 \mu \mathrm{M}$. Panels D-F show the stack of ${ }^{1} \mathrm{H}-\mathrm{NMR}$ spectra superimposed from the same T2-CPMG delay times with a constant compound concentration of $75 \mu \mathrm{M}$.

\section{6) Evidence for the detection of chemical exchange}

A range of compounds were further evaluated along with 1, 2, 3, etodolac, methylene blue and Evans blue (Table 1). Observed concentration for each compound was estimated by NMR both in buffer and solvent using an external standard reference. ${ }^{5}$ 
Table 1. Comparison of solution properties for a range of small molecules.

\begin{tabular}{|c|c|c|c|c|c|c|}
\hline \multirow[t]{2}{*}{ Compounds } & \multicolumn{2}{|c|}{$\begin{array}{c}\text { Observed } \\
\text { concentration }(\mu \mathrm{M})\end{array}$} & \multirow{2}{*}{$\begin{array}{c}\text { Peak area } \\
(800 \mathrm{~ms} / 1 \\
\mathrm{ms})(\%)\end{array}$} & \multirow[t]{2}{*}{ T2 (ms) } & \multirow[t]{2}{*}{$D\left(\mathrm{~m}^{2} / \mathrm{s}\right)$} & \multirow[t]{2}{*}{ Descriptor } \\
\hline & Buffer & Solvent & & & & \\
\hline L-tryptophan & 406 & 531 & 71 & 2405 & $4.56 \mathrm{E}-10$ & \\
\hline Etodolac & 428 & 375 & 60 & 1446 & $3.94 \mathrm{E}-10$ & \\
\hline Tartrazine & 295 & 284 & 52 & 1267 & $3.2 \mathrm{E}-10$ & Non-aggregators \\
\hline 1 & 305 & 379 & 52 & 1225 & $5.87 \mathrm{E}-10$ & \\
\hline Imidazole & 483 & 369 & 51 & 1109 & $8.4 \mathrm{E}-10$ & \\
\hline Thiamine & 373 & $308^{*}$ & 44 & 1008 & $3.56 \mathrm{E}-10$ & \\
\hline Lansoprazole & 390 & 393 & 41 & 1070 & $3.21 \mathrm{E}-10$ & Intermediate behaviors \\
\hline Gefitinib & 267 & 381 & 31 & 780 & $1.56 \mathrm{E}-10$ & \\
\hline Erythrosin B & 330 & 329 & 24 & 551 & $2.75 \mathrm{E}-10$ & \\
\hline Chlorpromazine & 345 & 391 & 11 & 362 & 3.66E-10 & \\
\hline Light green SF yellowish & 230 & 165 & 10 & 356 & $2.39 \mathrm{E}-10$ & \\
\hline Acid blue 9 & 275 & 316 & 9 & 357 & $2.24 \mathrm{E}-10$ & \\
\hline Flutamide & 378 & 436 & 7 & 305 & $4.02 \mathrm{E}-10$ & \\
\hline 3 & 347 & 399 & 6 & 61 & $5.33 \mathrm{E}-10$ & Aggregators \\
\hline 2 & 380 & 265 & 0 & 116 & $5.98 \mathrm{E}-10$ & \\
\hline Evans blue & 188 & 179 & 0 & 34 & 3.27E-12 & \\
\hline Imatinib & 191 & 333 & 11 & 334 & $2.61 \mathrm{E}-10$ & \\
\hline Methylene blue & 274 & $395^{\star}$ & 1 & 130 & $3.18 \mathrm{E}-10$ & \\
\hline Allura red & 284 & 361 & 0 & 121 & $1.95 \mathrm{E}-10$ & \\
\hline
\end{tabular}

Nominal concentration was $300 \mu \mathrm{M}$. Compounds with remaining T2-CPMG signal intensity below $25 \%$, between $25 \%$ and $50 \%$ and above $\mathbf{5 0 \%}$ were classified as aggregators, intermediate behaviors and non-aggregators, respectively.

Buffer: $50 \mathrm{mM} \mathrm{NaPi}, 100 \mathrm{mM} \mathrm{NaCl}, 100 \% \mathrm{D}_{2} \mathrm{O}$, pH 7.4. Solvent: 1:1 DMSO-d $\mathrm{d}_{6}$ and ACN- $\mathrm{d}_{3}$.

${ }^{*}$ Due to poor solubility in DMSO-ACN, the solvent used for these samples was $100 \%$ DMSO-d . $_{\text {. }}$

Measured peak area percentages span a wide range of values and when combined with solubility data, allows for a separation of these molecules into three categories: 1) nonaggregators, 2) intermediate behaviors, and 3) aggregators. Interestingly, the majority of compounds in the last two categories have previously been reported as aggregators or as having promiscuous behaviors. ${ }^{4,6-9}$

T2 relaxation times and diffusion coefficients were determined for all samples using T2-CPMG and diffusion-ordered spectroscopy (DOSY) NMR experiments, respectively. A comparison was done to determine if diffusion values corroborate the measurements of T2. DOSY provides a measure of translational diffusion and can therefore provide information on molecular size and 
shape, as well as dynamics. ${ }^{10-13}$ Comparisons between T2-CPMG and diffusion coefficients versus molecular weight of the compounds primary structures are shown in Figures S5 and S6, respectively. Overall correlations can be noted, especially when considering theoretical versus measured diffusion data as shown in Figure 5.

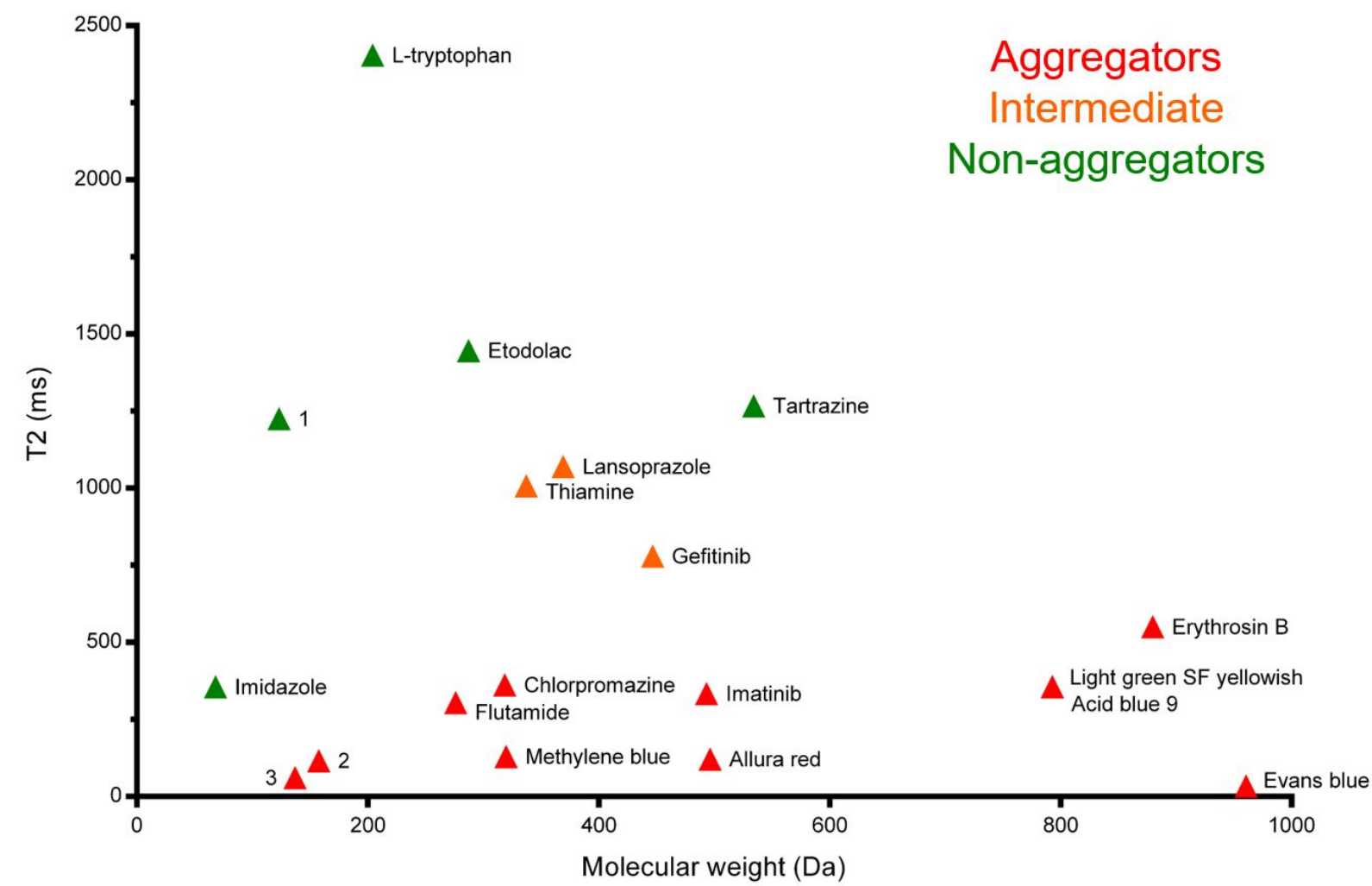

Figure S5. Correlation between T2 relaxation and molecular weight. While there is an expected correlation between T2 and molecular weight, chemical exchange processes also contribute to transverse relaxation rates. Therefore, compounds undergoing chemical exchange between states would exhibit shorter T2 relaxation times than compounds not undergoing exchange processes, as shown. Furthermore, compound aggregates are of larger size and would also exhibit shorter transverse relaxation times than freely soluble species. It is important to note that the molecular weights shown above are those expected for the free compounds. Aggregators therefore have T2 relaxation times that are much shorter than would be expected for the free compounds. Experimental points were colored according to their loss in T2-CPMG signal intensity between 1 and 800 ms delays (red, more than 75\% loss in signal intensity; orange, between 50\% and $75 \%$; green, less than $50 \%$ ).

In contrast, the T2-CPMG experiment is able to indirectly detect the presence of some very large "NMR-invisible" aggregates. The chemical exchange contribution to T2 resulting from 
exchange between single molecules and aggregated species of various sizes is therefore a more appropriate reporter of self-association and aggregation.

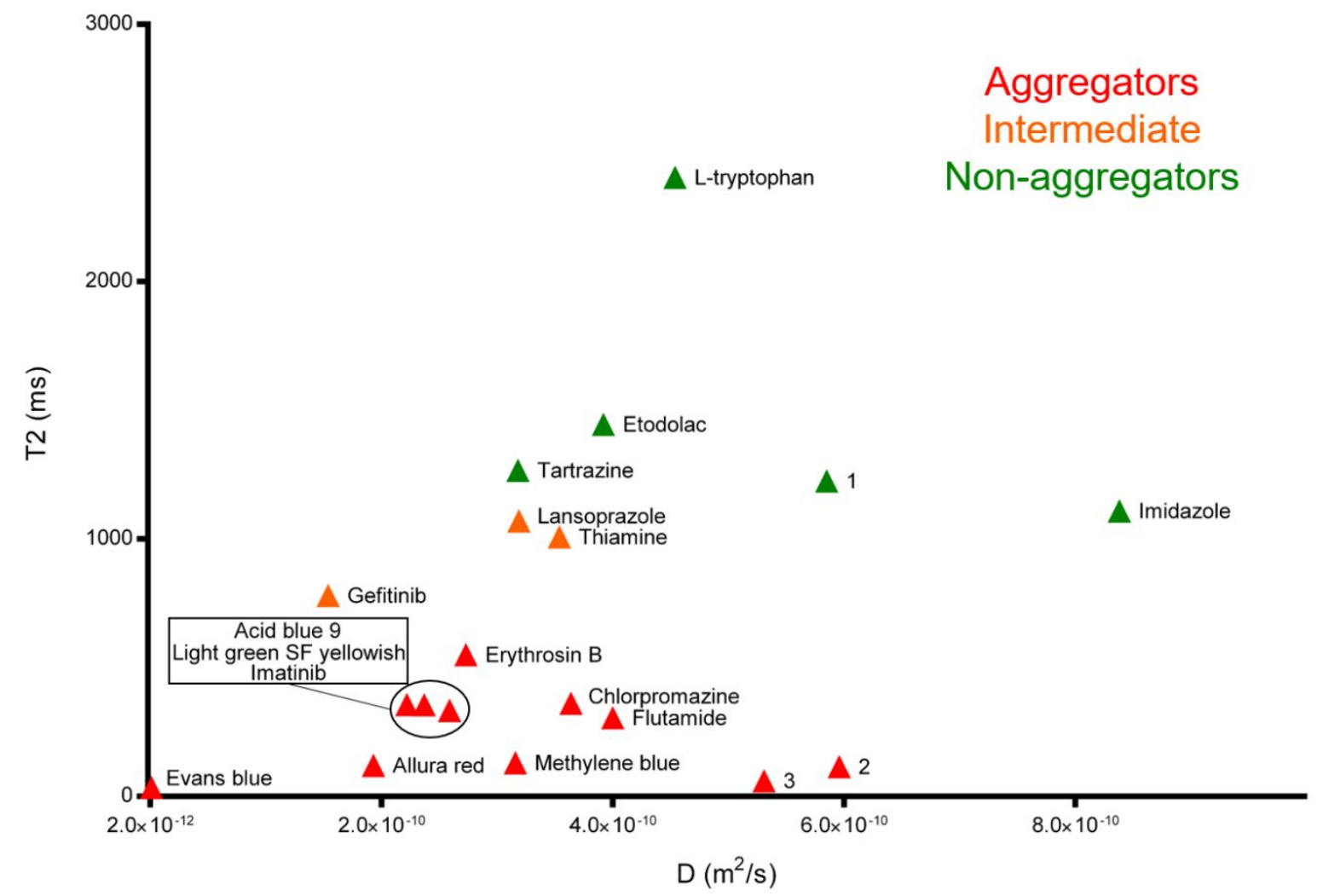

Figure S6. There is only modest correlation between T2 and diffusion coefficient. As mentioned previously, this can be explained by chemical exchange contributions to T2. While diffusion is sensitive primarily to molecular size and has a detection range limited to smaller species that are detectable by NMR, T2 can elucidate very large "NMR invisible" entities indirectly via chemical exchange. It is possible that the diffusion coefficients measured for aggregators only represents the "NMR visible" fraction, thereby misrepresenting the true size distribution of aggregate species in the sample. Experimental points were colored according to their loss in T2-CPMG signal intensity between 1 and $800 \mathrm{~ms}$ delays (red, more than $75 \%$ loss in signal intensity; orange, between $50 \%$ and $75 \%$; green, less than $50 \%$ ).

\section{7) Consistency between the use of T2 and peak area percentages}

Figures 6, 7 and S3 illustrate that only two T2-CPMG delays can be used to rapidly assess compound solution behaviors. This can prove very useful in reducing NMR acquisition time when curating large compound libraries. Figure S7 illustrates the correlation between the T2 and peak area calculation for all compounds present in Table 1. Peak areas were measured using two delay times as described above, while the T2 relaxation times were obtained by fitting across eight T2CPMG delays. Therefore, peak areas can serve as reliable and fast filters in order to quickly curate large numbers of compounds. 


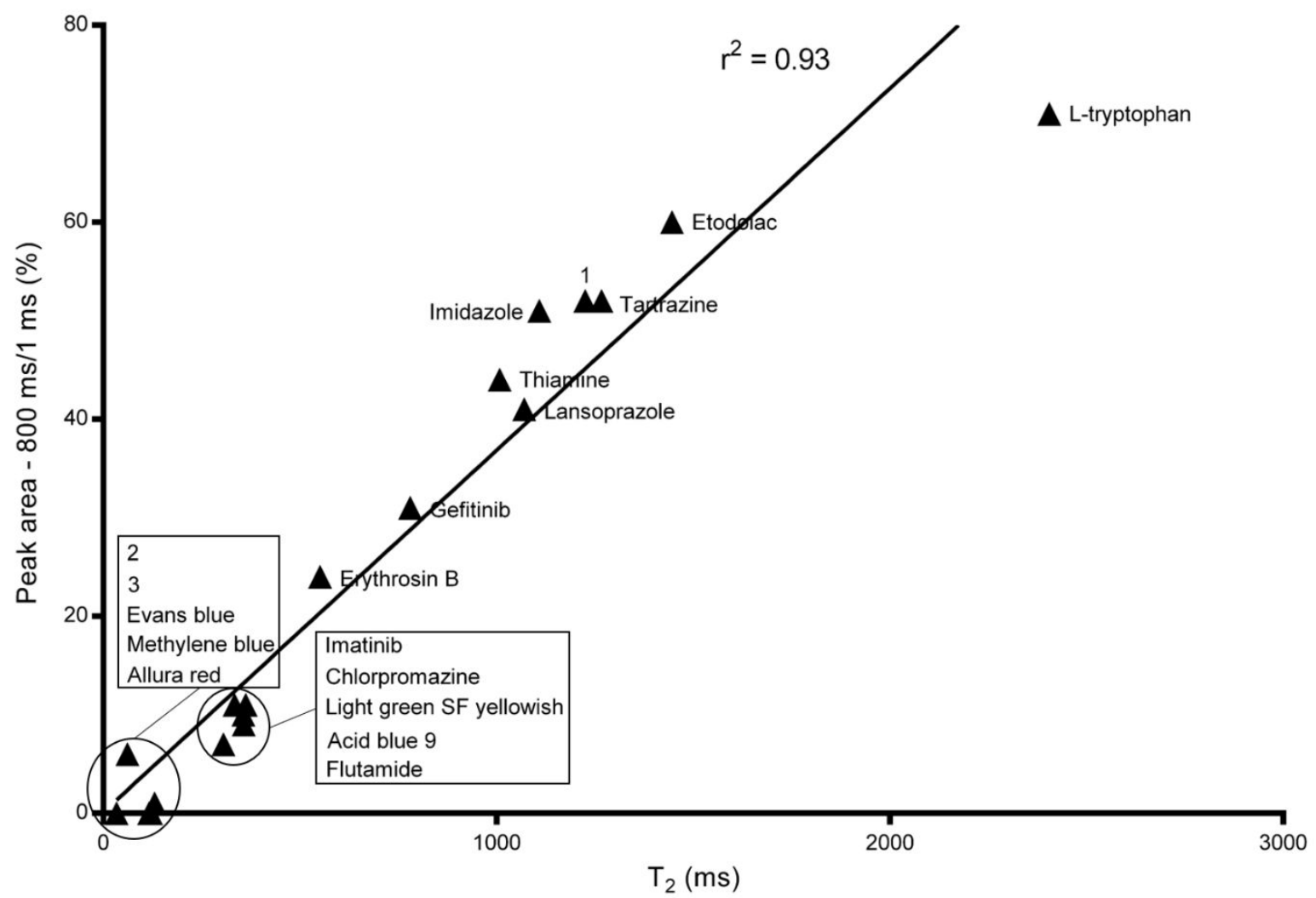

Figure S7. Percentages of peak area for 800 versus 1 milliseconds delays correlate well with the T2 relaxation times obtained by fitting several delay points.

\section{8) Compound information}

\begin{tabular}{|c|c|c|c|c|c|}
\hline Compound Name & SMILES & Supplier & Catalog No. & CAS & $\begin{array}{c}\text { In this } \\
\text { manuscript }\end{array}$ \\
\hline $\begin{array}{c}\text { Light green SF } \\
\text { yellowish }\end{array}$ & $\begin{array}{c}\mathrm{CCN}(\mathrm{CC} 1=\mathrm{CC}(=\mathrm{CC} \\
=\mathrm{C} 1) \mathrm{S}(=\mathrm{O})(=\mathrm{O})[\mathrm{O}- \\
]) \mathrm{C} 2=\mathrm{CC}=\mathrm{C}(\mathrm{C}=\mathrm{C} 2) \mathrm{C} \\
(=\mathrm{C} 3 \mathrm{C}=\mathrm{CC}(=[\mathrm{N}+](\mathrm{C} \\
\mathrm{C}) \mathrm{CC} 4=\mathrm{CC}(=\mathrm{CC}=\mathrm{C} 4 \\
) \mathrm{S}(=\mathrm{O})(=\mathrm{O})[\mathrm{O}- \\
]) \mathrm{C}=\mathrm{C} 3) \mathrm{C} 5=\mathrm{CC}=\mathrm{C}(\mathrm{C} \\
=\mathrm{C} 5) \mathrm{S}(=\mathrm{O})(=\mathrm{O})[\mathrm{O}- \\
\text { ]. }[\mathrm{Na}+] \cdot[\mathrm{Na}+]\end{array}$ & Alfa Aesar & B23330 & $5141-20-8$ & $\begin{array}{c}\text { Figures } 1,5, \text { S5- } \\
\text { S7, Table } 1\end{array}$ \\
\hline
\end{tabular}




\begin{tabular}{|c|c|c|c|c|c|}
\hline Evans Blue & $\begin{array}{c}C C 1=C(C=C C(=C 1) \\
C 2=C C(=C(C=C 2) N \\
=N C 3=C(C 4=C(C=C \\
3) C(=C C(=C 4 N) S(= \\
O)(=O)[O- \\
\text { ])S }(=O)(=O)[O- \\
\text { ])O }) \mathrm{C}) N=N C 5=C(C 6 \\
=C(C=C 5) C(=C C(=C \\
6 N) S(=O)(=O)[O- \\
\text { ])S }(=O)(=O)[O- \\
\text { ])O. } \\
{[\mathrm{Na}+] \cdot[\mathrm{Na}+] \cdot[\mathrm{Na}+} \\
] \cdot[\mathrm{Na}+]\end{array}$ & Sigma & E2129 & $314-13-6$ & $\begin{array}{c}\text { Figures } 1,4,5, \\
\text { S1, S4-S7, Table } \\
1\end{array}$ \\
\hline Acid violet 49 & $\begin{array}{c}C C N(C C 1=C C(=C C \\
=C 1) S(=O)(=O) O) C 2 \\
=C C=C(C=C 2) C(=C \\
3 C=C C(=[N+](C) C) C \\
=C 3) C 4=C C=C(C=C \\
4) N(C C) C C 5=C C(=C \\
C=C 5) S(=O)(=O)[O- \\
] \cdot[N a+]\end{array}$ & $\mathrm{TCl}$ & 42640 & $1694-09-3$ & Figure 1 \\
\hline Pranlukast & $\begin{array}{c}C 1=C C=C(C=C 1) C C \\
C C O C 2=C C=C(C=C \\
2) C(=O) N C 3=C C=C \\
C 4=C 3 O C(=C C 4=O) \\
C 5=N N N=N 5\end{array}$ & Beta Pharma & $56-05418$ & $103177-37-3$ & Figure 1 \\
\hline $\begin{array}{c}\text { 2-methyl-1,3(2H,4H)- } \\
\text { isoquinolinedione }\end{array}$ & $\begin{array}{c}\mathrm{CN} 1 \mathrm{C}(=\mathrm{O}) \mathrm{CC} 2=\mathrm{CC}= \\
\mathrm{CC}=\mathrm{C} 2 \mathrm{C} 1=\mathrm{O}\end{array}$ & Key Organics & 10L-733 & $4494-53-5$ & Figure 2 \\
\hline Etodolac & $\begin{array}{c}\mathrm{CCC} 1=\mathrm{CC}=\mathrm{CC} 2=\mathrm{C} 1 \\
\mathrm{NC} 3=\mathrm{C} 2 \mathrm{CCOC} 3(\mathrm{CC} \\
) \mathrm{CC}(=\mathrm{O}) \mathrm{O}\end{array}$ & Sigma & E0516 & $41340-25-4$ & $\begin{array}{c}\text { Figures } 4,5, \mathrm{~S} 1 \text {, } \\
\text { S4-S7, Table } 1\end{array}$ \\
\hline Methylene Blue & $\begin{array}{c}\mathrm{CN}(\mathrm{C}) \mathrm{C} 1=\mathrm{CC} 2=\mathrm{C}(\mathrm{C} \\
=\mathrm{C} 1) \mathrm{N}=\mathrm{C} 3 \mathrm{C}=\mathrm{CC}(=[ \\
\mathrm{N}+](\mathrm{C}) \mathrm{C}) \mathrm{C}=\mathrm{C} 3 \mathrm{~S} 2 \cdot[\mathrm{Cl} \\
-]\end{array}$ & Sigma & M9140 & $122965-43-9$ & $\begin{array}{c}\text { Figures } 4,5, \mathrm{~S} 1 \text {, } \\
\text { S4-S7, Table } 1\end{array}$ \\
\hline
\end{tabular}




\begin{tabular}{|c|c|c|c|c|c|}
\hline Nicotinic acid (1) & $\begin{aligned} C 1=C C( & =C N=C 1) C( \\
& =O) O\end{aligned}$ & Key Organics & PS-4255 & $59-67-6$ & $\begin{array}{c}\text { Figures 5, 6, 9, } \\
\text { S2, S5-S7, Table } \\
1\end{array}$ \\
\hline $\begin{array}{l}\text { 5-chloro-2- } \\
\text { pyridinecarboxylic } \\
\text { acid (2) }\end{array}$ & $\begin{array}{c}\mathrm{C} 1=\mathrm{CC}(=\mathrm{NC}=\mathrm{C} 1 \mathrm{Cl}) \\
\mathrm{C}(=\mathrm{O}) \mathrm{O}\end{array}$ & Key Organics & BB-0607 & $86873-60-1$ & $\begin{array}{c}\text { Figures 5, 6, 9, } \\
\text { S2, S5-S7, Table } \\
1\end{array}$ \\
\hline $\begin{array}{l}\text { 3-methylpyridine-2- } \\
\text { carboxylic acid (3) }\end{array}$ & $\begin{array}{c}C C 1=C(N=C C=C 1) C \\
(=O) O\end{array}$ & Key Organics & PS-3239 & $4021-07-2$ & $\begin{array}{l}\text { Figures } 5,6, \text { S2, } \\
\text { S5-S7, Table } 1\end{array}$ \\
\hline $\begin{array}{l}\text { (1-methyl-1H- } \\
\text { benzimidazol-2- } \\
\text { yl)methanol (4) }\end{array}$ & $\begin{aligned} \mathrm{CN} 1 \mathrm{C} 2 & =\mathrm{CC}=\mathrm{CC}=\mathrm{C} 2 \\
\mathrm{~N} & =\mathrm{C} 1 \mathrm{CO}\end{aligned}$ & Chembridge & 4900605 & $7467-35-8$ & Figure 7 \\
\hline $\begin{array}{l}\text { N-methyl-1-(1- } \\
\text { methyl-1H- } \\
\text { benzimidazol-2- } \\
\text { yl)methanamine } \\
\text { dihydrochloride (5) }\end{array}$ & $\begin{array}{c}\mathrm{CNCC} 1=\mathrm{NC} 2=\mathrm{CC}=\mathrm{C} \\
\mathrm{C}=\mathrm{C} 2 \mathrm{~N} 1 \mathrm{C} \cdot \mathrm{Cl} . \mathrm{Cl}\end{array}$ & Chembridge & 4015772 & $\mathrm{~N} / \mathrm{A}$ & Figure 7 \\
\hline $\begin{array}{l}\text { [(1-methyl-1H- } \\
\text { benzimidazol-2- } \\
\text { yl)methyl]amine } \\
\text { hydrochloride (6) }\end{array}$ & $\begin{aligned} \mathrm{CN} 1 \mathrm{C} 2 & =\mathrm{CC}=\mathrm{CC}=\mathrm{C} 2 \\
\mathrm{~N} & =\mathrm{C} 1 \mathrm{CN}\end{aligned}$ & Chembridge & 4100112 & $\mathrm{~N} / \mathrm{A}$ & Figure 7 \\
\hline $\begin{array}{c}\text { [2-(1-methyl-1H- } \\
\text { benzimidazol-2- } \\
\text { yl)ethyl]amine } \\
\text { dihydrochloride (7) }\end{array}$ & $\begin{array}{c}\mathrm{CN} 1 \mathrm{C} 2=\mathrm{CC}=\mathrm{CC}=\mathrm{C} 2 \\
\mathrm{~N}=\mathrm{C} 1 \mathrm{CCN} \cdot \mathrm{Cl} . \mathrm{Cl}\end{array}$ & Chembridge & 4100113 & $\mathrm{~N} / \mathrm{A}$ & Figure 7 \\
\hline $\begin{array}{l}\text { [3-(1-methyl-1H- } \\
\text { benzimidazol-2- } \\
\text { yl)propyl]amine } \\
\text { dihydrochloride } \\
\text { hydrate (8) }\end{array}$ & $\begin{array}{l}\mathrm{CN} 1 \mathrm{C} 2=\mathrm{CC}=\mathrm{CC}=\mathrm{C} 2 \\
\mathrm{~N}=\mathrm{C} 1 \mathrm{CCCN} . \mathrm{O} . \mathrm{Cl} . \mathrm{Cl}\end{array}$ & Chembridge & 4033392 & $\mathrm{~N} / \mathrm{A}$ & Figure 7 \\
\hline $\begin{array}{c}\text { 6-methyl-1,2,3,4- } \\
\text { tetrahydro-11H- } \\
\text { pyrido[2,1- } \\
\text { b]quinazolin-11-one } \\
\text { (9) }\end{array}$ & $\begin{array}{c}\mathrm{CC} 1=\mathrm{CC}=\mathrm{CN} 2 \mathrm{C}(=\mathrm{O}) \\
\mathrm{C} 3=\mathrm{C}(\mathrm{CCCC} 3) \mathrm{N}=\mathrm{C} 1 \\
2\end{array}$ & Chembridge & 9221258 & $\mathrm{~N} / \mathrm{A}$ & Figure 8 \\
\hline
\end{tabular}




\begin{tabular}{|c|c|c|c|c|c|}
\hline $\begin{array}{c}\text { 6-hydroxy-1,2,3,4- } \\
\text { tetrahydro-11H- } \\
\text { pyrido[2,1- } \\
\text { b]quinazolin-11-one } \\
\text { (10) }\end{array}$ & $\begin{array}{c}\mathrm{OC} 1=\mathrm{CC}=\mathrm{CN} 2 \mathrm{C}(=\mathrm{O} \\
\mathrm{C} 3=\mathrm{C}(\mathrm{CCCC} 3) \mathrm{N}=\mathrm{C} \\
12\end{array}$ & Chembridge & 9200099 & $\mathrm{~N} / \mathrm{A}$ & Figure 8 \\
\hline $\begin{array}{l}\text { 3-ethyl-9-hydroxy-2- } \\
\text { methyl-4H- } \\
\text { pyrido[1,2- } \\
\text { a]pyrimidin-4-one } \\
\text { (11) }\end{array}$ & $\begin{array}{c}\mathrm{CCC} 1=\mathrm{C}(\mathrm{N}=\mathrm{C} 2 \mathrm{C}(=\mathrm{C} \\
\mathrm{C}=\mathrm{CN} 2 \mathrm{C} 1=\mathrm{O}) \mathrm{O}) \mathrm{C}\end{array}$ & Chembridge & 9226831 & $\mathrm{~N} / \mathrm{A}$ & Figure 8 \\
\hline $\begin{array}{l}\text { 3-ethyl-2,6-dimethyl- } \\
\text { 4H-pyrido[1,2- } \\
\text { a]pyrimidin-4-one } \\
\text { (12) }\end{array}$ & $\begin{array}{l}C C C 1=C(N=C 2 C=C \\
C=C(N 2 C 1=O) C) C\end{array}$ & Chembridge & 9205478 & $\mathrm{~N} / \mathrm{A}$ & Figure 8 \\
\hline $\begin{array}{l}\text { 3-ethyl-2-methyl-4H- } \\
\text { pyrido[1,2- } \\
\text { a]pyrimidin-4-one } \\
\text { (13) }\end{array}$ & $\begin{array}{c}C C C 1=C(N=C 2 C=C \\
C=C N 2 C 1=O) C\end{array}$ & Chembridge & 9281741 & $\mathrm{~N} / \mathrm{A}$ & Figure 8 \\
\hline $\begin{array}{c}\text { 3-(4- } \\
\text { bromophenoxy)prop } \\
\text { anenitrile (14) }\end{array}$ & 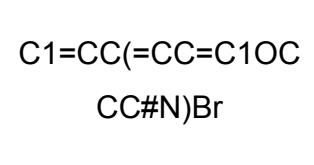 & Key Organics & PS-3130 & $118449-57-3$ & Figure 10 \\
\hline $\begin{array}{c}\text { [(6-methoxy-1H- } \\
\text { benzimidazol-2- } \\
\text { yl)methyl]amine } \\
\text { dihydrochloride (15) }\end{array}$ & $\begin{array}{l}\text { Cl.Cl.COc1ccc2nc(C } \\
\text { N) }[\mathrm{nH}] \mathrm{c} 2 \mathrm{c} 1\end{array}$ & Chembridge & 4101295 & N/A & Figure S3 \\
\hline $\begin{array}{c}\text { [(6-chloro-1H- } \\
\text { benzimidazol-2- } \\
\text { yl)methyl]amine } \\
\text { dihydrochloride (16) }\end{array}$ & $\begin{array}{l}\text { Cl.Cl.NCc1nc2 } \operatorname{ccc}(\mathrm{Cl} \\
) \mathrm{cc} 2[\mathrm{nH}] 1\end{array}$ & Chembridge & 4101293 & N/A & Figure S3 \\
\hline $\begin{array}{c}\text { [(6-fluoro-1H- } \\
\text { benzimidazol-2- } \\
\text { yl)methyl]methylamin } \\
\text { e dihydrochloride } \\
\text { (17) }\end{array}$ & $\begin{array}{l}\text { Cl.Cl.CNCc1nc2ccc( } \\
\text { F) } \operatorname{cc} 2[\mathrm{nH}] 1\end{array}$ & Chembridge & 4004431 & $\mathrm{~N} / \mathrm{A}$ & Figure S3 \\
\hline
\end{tabular}




\begin{tabular}{|c|c|c|c|c|c|}
\hline $\begin{array}{c}\text { [(5-fluoro-1H- } \\
\text { benzimidazol-2- } \\
\text { yl)methyl]amine } \\
\text { dihydrochloride (18) }\end{array}$ & $\begin{array}{c}\mathrm{C} 1=\mathrm{CC} 2=\mathrm{C}(\mathrm{C}=\mathrm{C} 1 \mathrm{~F}) \\
\mathrm{NC}(=\mathrm{N} 2) \mathrm{CN} . \mathrm{Cl} . \mathrm{Cl}\end{array}$ & Chembridge & 4002683 & $\mathrm{~N} / \mathrm{A}$ & Figure S3 \\
\hline $\begin{array}{c}\text { [2-(5-fluoro-1H- } \\
\text { benzimidazol-2- } \\
\text { yl)ethyl]methylamine } \\
\text { dihydrochloride (19) }\end{array}$ & $\begin{array}{l}C N C C C 1=N C 2=C(N \\
1) C=C(C=C 2) F . C l . C l\end{array}$ & Chembridge & 4009689 & N/A & Figure S3 \\
\hline Imidazole & $\mathrm{C} 1=\mathrm{CN}=\mathrm{CN} 1$ & Sigma & 12399 & $288-32-4$ & $\begin{array}{c}\text { Figures 5, S5- } \\
\text { S7, Table } 1\end{array}$ \\
\hline Lansoprazole & $\begin{array}{c}C C 1=C(C=C N=C 1 C \\
S(=O) C 2=N C 3=C C= \\
C C=C 3 N 2) O C C(F)(F \\
) F\end{array}$ & Sigma & L8533 & $103577-45-3$ & $\begin{array}{c}\text { Figures 5, S5- } \\
\text { S7, Table } 1\end{array}$ \\
\hline Tartrazine & $\begin{array}{c}\mathrm{C} 1=\mathrm{CC}(=\mathrm{CC}=\mathrm{C} 1 \mathrm{~N}= \\
\mathrm{NC} 2 \mathrm{C}(=\mathrm{NN}(\mathrm{C} 2=\mathrm{O}) \mathrm{C} \\
3=\mathrm{CC}=\mathrm{C}(\mathrm{C}=\mathrm{C} 3) \mathrm{S}(= \\
\mathrm{O})(=\mathrm{O})[\mathrm{O}-]) \mathrm{C}(=\mathrm{O})[\mathrm{O}- \\
]) \mathrm{S}(=\mathrm{O})(=\mathrm{O})[\mathrm{O}- \\
] .[\mathrm{Na}+] \cdot[\mathrm{Na}+] \cdot[\mathrm{Na}+]\end{array}$ & Alfa Aesar & A17682 & $1934-21-0$ & $\begin{array}{c}\text { Figures 5, S5- } \\
\text { S7, Table } 1\end{array}$ \\
\hline Thiamine & $\begin{array}{c}C C 1=C(S C=[N+] 1 C \\
C 2=C N=C(N=C 2 N) C \\
) C C O\end{array}$ & Sigma & T1270 & $67-03-8$ & $\begin{array}{c}\text { Figures 5, S5- } \\
\text { S7, Table } 1\end{array}$ \\
\hline L-tryptophan & $\begin{array}{c}\mathrm{C} 1=\mathrm{CC}=\mathrm{C} 2 \mathrm{C}(=\mathrm{C} 1) \mathrm{C} \\
(=\mathrm{CN} 2) \mathrm{CC}(\mathrm{C}(=\mathrm{O}) \mathrm{O}) \\
\mathrm{N}\end{array}$ & RPI & T60080 & $73-22-3$ & $\begin{array}{c}\text { Figures 5, S5- } \\
\text { S7, Table } 1\end{array}$ \\
\hline Erythrosin B & $\begin{array}{l}\mathrm{C} 1=\mathrm{CC}=\mathrm{C} 2 \mathrm{C}(=\mathrm{C} 1) \mathrm{C} \\
(=\mathrm{O}) \mathrm{OC} 23 \mathrm{C} 4=\mathrm{CC}(= \\
\mathrm{C}(\mathrm{C}(=\mathrm{C} 4 \mathrm{OC} 5=\mathrm{C}(\mathrm{C}(= \\
\mathrm{C}(\mathrm{C}=\mathrm{C} 35) \mathrm{I}) \mathrm{O}) \mathrm{I}) \mathrm{I}) \mathrm{O}) \mathrm{I}\end{array}$ & $\mathrm{TCl}$ & T0557 & $16423-68-0$ & $\begin{array}{c}\text { Figures 5, S5- } \\
\text { S7, Table } 1\end{array}$ \\
\hline Gefitinib & $\begin{array}{c}\mathrm{COC} 1=\mathrm{C}(\mathrm{C}=\mathrm{C} 2 \mathrm{C}(=\mathrm{C} \\
1) \mathrm{N}=\mathrm{CN}=\mathrm{C} 2 \mathrm{NC} 3=\mathrm{C} \\
\mathrm{C}(=\mathrm{C}(\mathrm{C}=\mathrm{C} 3) \mathrm{F}) \mathrm{Cl}) \mathrm{OC} \\
\mathrm{CCN} 4 \mathrm{CCOCC} 4\end{array}$ & Beta Pharma & $86-33451$ & $184475-35-2$ & $\begin{array}{c}\text { Figures 5, S5- } \\
\text { S7, Table } 1\end{array}$ \\
\hline
\end{tabular}




\begin{tabular}{|c|c|c|c|c|c|}
\hline Acid blue 9 & $\begin{array}{c}\mathrm{CCN}(\mathrm{CC} 1=\mathrm{CC}(=\mathrm{CC} \\
=\mathrm{C} 1) \mathrm{S}(=\mathrm{O})(=\mathrm{O})[\mathrm{O}- \\
]) \mathrm{C} 2=\mathrm{CC}=\mathrm{C}(\mathrm{C}=\mathrm{C} 2) \mathrm{C} \\
(=\mathrm{C} 3 \mathrm{C}=\mathrm{CC}(=[\mathrm{N}+](\mathrm{C} \\
\mathrm{C}) \mathrm{CC} 4=\mathrm{CC}(=\mathrm{CC}=\mathrm{C} 4 \\
) \mathrm{S}(=\mathrm{O})(=\mathrm{O})[\mathrm{O}- \\
]) \mathrm{C}=\mathrm{C} 3) \mathrm{C} 5=\mathrm{CC}=\mathrm{CC} \\
=\mathrm{C} 5 \mathrm{~S}(=\mathrm{O})(=\mathrm{O})[\mathrm{O}- \\
] .[\mathrm{NH} 4++] \cdot[\mathrm{NH} 4+]\end{array}$ & $\mathrm{TCl}$ & B0790 & $3844-45-9$ & $\begin{array}{c}\text { Figures 5, S5- } \\
\text { S7, Table } 1\end{array}$ \\
\hline Chlorpromazine & $\begin{array}{l}\mathrm{CN}(\mathrm{C}) \mathrm{CCCN} 1 \mathrm{C} 2=\mathrm{C} \\
\mathrm{C}=\mathrm{CC}=\mathrm{C} 2 \mathrm{SC} 3=\mathrm{C} 1 \mathrm{C} \\
=\mathrm{C}(\mathrm{C}=\mathrm{C} 3) \mathrm{Cl}\end{array}$ & AK Scientific & M176 & $50-53-3$ & $\begin{array}{c}\text { Figures 5, S5- } \\
\text { S7, Table } 1\end{array}$ \\
\hline Flutamide & $\begin{array}{c}\mathrm{CC}(\mathrm{C}) \mathrm{C}(=\mathrm{O}) \mathrm{NC} 1=\mathrm{C} \\
\mathrm{C}(=\mathrm{C}(\mathrm{C}=\mathrm{C} 1)[\mathrm{N}+](=\mathrm{O} \\
)[\mathrm{O}-]) \mathrm{C}(\mathrm{F})(\mathrm{F}) \mathrm{F}\end{array}$ & Sigma & F9397 & $13311-84-7$ & $\begin{array}{c}\text { Figures 5, S5- } \\
\text { S7, Table } 1\end{array}$ \\
\hline Allura red & $\begin{array}{c}\mathrm{CC} 1=\mathrm{CC}(=\mathrm{C}(\mathrm{C}=\mathrm{C} 1 \mathrm{~S} \\
(=\mathrm{O})(=\mathrm{O})[\mathrm{O}- \\
\text { ])OC }) \mathrm{N}=\mathrm{NC} 2=\mathrm{C}(\mathrm{C}= \\
\mathrm{CC}=\mathrm{C} 2 \mathrm{C}=\mathrm{CC}(=\mathrm{C} 3) \\
\mathrm{S}(=\mathrm{O})(=\mathrm{O})[\mathrm{O}- \\
\text { ])O.[Na+].[Na+] }\end{array}$ & $\mathrm{TCl}$ & A0943 & $25956-17-6$ & $\begin{array}{c}\text { Figures 5, S5- } \\
\text { S7, Table } 1\end{array}$ \\
\hline Imatinib & $\begin{array}{c}C C 1=C(C=C(C=C 1) \\
N C(=O) C 2=C C=C(C \\
=C 2) C N 3 C C N(C C 3) \\
C) N C 4=N C=C C(=N 4 \\
) C 5=C N=C C=C 5\end{array}$ & Beta Pharma & $86-33437$ & 152459-95-5 & $\begin{array}{c}\text { Figures 5, S5- } \\
\text { S7, Table } 1\end{array}$ \\
\hline
\end{tabular}




\section{References}

1. Brown, K. C., Essential Mathematics for NMR and MRI Spectroscopists. Royal Society of Chemistry: 2016.

2. LaPlante, S. R.; Carson, R.; Gillard, J.; Aubry, N.; Coulombe, R.; Bordeleau, S.; Bonneau, P.; Little, M.; O'Meara, J.; Beaulieu, P. L., Compound Aggregation in Drug Discovery: Implementing a Practical NMR Assay for Medicinal Chemists. J. Med. Chem. 2013, 56 (12), 5142-5150.

3. LaPlante, S. R.; Aubry, N.; Bolger, G.; Bonneau, P.; Carson, R.; Coulombe, R.; Sturino, C.; Beaulieu, P. L., Monitoring Drug Self-Aggregation and Potential for Promiscuity in Off-Target In Vitro Pharmacology Screens by a Practical NMR Strategy. J. Med. Chem. 2013, 56 (17), 7073-7083.

4. Murugesan, J. R.; Shahout, F.; Dlim, M.; Langella, M. M.; Cuadra-Foy, E.; Forgione, P.; LaPlante, S. R., Revealing Dye and Dye-drug Aggregation Into Nano-entities using NMR. Dyes Pigm. 2018, 153, 300306.

5. Akoka, S.; Barantin, L.; Trierweiler, M., Concentration Measurement by Proton NMR Using the ERETIC Method. Anal. Chem. 1999, 71 (13), 2554-2557.

6. Doak, A. K.; Wille, H.; Prusiner, S. B.; Shoichet, B. K., Colloid Formation by Drugs in Simulated Intestinal Fluid. J. Med. Chem. 2010, 53 (10), 4259-4265.

7. Schreier, S.; Malheiros, S. V. P.; de Paula, E., Surface Active Drugs: Self-association and Interaction with Membranes and Surfactants. Physicochemical and Biological Aspects. Biochim. Biophys. Acta 2000, 1508 (1), 210-234.

8. Ganesan, L.; Buchwald, P., The Promiscuous Protein Binding Ability of Erythrosine B Studied by Metachromasy (metachromasia). J. Mol. Recognit. 2013, 26 (4), 181-9.

9. Owen, S. C.; Doak, A. K.; Wassam, P.; Shoichet, M. S.; Shoichet, B. K., Colloidal Aggregation Affects the Efficacy of Anticancer Drugs in Cell Culture. ACS Chem. Biol. 2012, 7 (8), 1429-1435.

10. Šmejkalová, D.; Piccolo, A., Aggregation and Disaggregation of Humic Supramolecular Assemblies by NMR Diffusion Ordered Spectroscopy (DOSY-NMR). Environ. Sci. Technol. 2008, 42 (3), 699-706.

11. Neufeld, R.; Stalke, D., Accurate Molecular Weight Determination of Small Molecules Via DOSY-NMR by Using External Calibration Curves with Normalized Diffusion Coefficients. Chem. Sci. 2015, 6 (6), 3354-3364.

12. Simpson, A. J., Determining the Molecular Weight, Aggregation, Structures and Interactions of Natural Organic Matter Using Diffusion Ordered Spectroscopy. Magn. Reson. Chem. 2002, 40 (13), S72-S82.

13. Chen, A.; Johnson, C. S.; Lin, M.; Shapiro, M. J., Chemical Exchange in Diffusion NMR Experiments. J. Am. Chem. Soc. 1998, 120 (35), 9094-9095. 